\title{
Modeling RNA Topological Structures Using Small Angle X-Ray Scattering
}

Yuba R. Bhandari ${ }^{\mathrm{a}^{*}}$, Wei Jiang ${ }^{\mathrm{b}}$, Eric A. Stahlberg ${ }^{\mathrm{c}}$, Jason R. Stagno ${ }^{\mathrm{a}} \&$ Yun-Xing Wang ${ }^{\mathrm{a}}$

${ }^{a}$ Protein-Nucleic Acid Interaction Section, Structural Biophysics Laboratory, Center for Cancer Research, National Cancer Institute, National Institutes of Health, Frederick, Maryland 21702 USA; ${ }^{b}$ Argonne National Laboratory, Lemont, Illinois; ' $\mathrm{c}$ ata Science and Information Technology Program, Frederick National Laboratory for Cancer Research, Frederick, Maryland 21702 USA;

*Corresponding author: Email: bhandariyr@mail.nih.gov 


\begin{abstract}
Detailed understanding of the structure and function relationship of RNA requires knowledge about RNA three-dimensional (3D) topological folding. However, there are very few unique RNA entries in structure databases. This is due to challenges in determining 3D structures of RNA using conventional methods, such as X-ray crystallography and NMR spectroscopy, despite significant advances in both of these technologies. Computational methods have come a long way in accurately predicting the 3D structures of small ( $<50 \mathrm{nt})$ RNAs to within a few angstroms compared to their native folds. However, lack of an apparent correlation between an RNA primary sequence and its 3D fold ultimately limits the success of purely computational approaches. In this context, small angle X-ray scattering (SAXS) serves as a valuable tool by providing global shape information of RNA. In this article, we review the progress in determining RNA 3D topological structures, including a new method that combines secondary structural information and SAXS data to sample conformations generated through hierarchical moves of commonly observed RNA motifs.
\end{abstract}




\section{Keywords:}

SAXS; RNA; conformation; motif; moves; topological

\section{Abbreviations:}

SAXS, small angle X-ray scattering; PDB, protein data bank; nt, nucleotides; rmsd, root mean squared deviation; 3D, three dimensional; SASA, solvent accessible surface area. 


\section{Introduction}

RNAs play pivotal roles in many cellular processes, including the regulation of gene expression [1-3]. Knowledge of their 3D structures is vital to understanding their biological functions. Despite importance of RNA in biology, there is general lack of RNA structural information in the current databases, where there are fewer than 100 unique RNA structure entries, excluding simple RNA hairpins or duplexes. Clearly, this is not due to lack of interest in RNA structure and function. But rather, it is due to some technical hurdles. High-resolution techniques, such as X-ray crystallography and NMR spectroscopy, are ideal for studying macromolecular structures. However, when applied to RNAs, they face several technical limitations, such as obtaining crystals suitable for diffraction or excessive NMR-signal overlap.

The RNA folding problem is unique in many aspects. The electron-rich sugarphosphate backbone of RNAs makes their folding architecture susceptible to the surrounding polar environment [4]. Since they are composed of only four different nucleotide types and a limited number of secondary structural motifs, RNAs must be very flexible for adopting diverse conformations in order to meet functional needs. A single strand of RNA folds back on itself to form loops and duplexes, and duplexes across a junction may helically stack to give further stability to the molecule. Duplexes and loops from separate domains may participate in long-range packing interactions to drive 3D folding. Various computational and experimental methods for RNA structure determination have emerged to circumvent the limitations posed by conventional methods by exploiting the general structural constraints and folding principles derived from known structures in databases. Purely computational software [5-7], which solely 
utilize empirical force fields and database restraints, have shown that it is possible to predict the native folds of mostly smaller (<50 nt) RNAs. Nonetheless, structure prediction algorithms are limited by the availability of the folding architectures in the databases, and predicted models may deviate substantially from the native structures as the size of the molecule increases. Therefore, hybrid methods that incorporate experimental constraints and secondary structural information in computational modeling may provide a practical way to determine $3 \mathrm{D}$ topological structures of large RNA molecules [8], especially now that secondary structures of RNA can be predicted and verified by robust experimental means such as SHAPE technology $[9,10]$.

Small angle X-ray scattering (SAXS) is a well understood physical phenomenon [11]. An X-ray beam passes through a solution and elastically scatters after hitting molecules in the solution. The scattering profile is recoded and radially averaged to reduce to a one-dimensional curve of scattering intensity plotted as function of scattering angles (momentum transfer). The scattering profile for biomacromolecues in solution can be obtained by subtraction of the SAXS contribution from buffer solution. The shape of such a scattering curve contains structural information [11, 12]. For this reason, SAXS data is used to derive molecular parameters such as overall size, molecular weight, oligomeric state, and global shape (Figure 1) [12]. In studying RNA, SAXS is particularly useful for molecular modeling. First of all, RNAs are predominantly made up of A-form duplexes. The elongated and modular nature of duplexes makes it relatively easy to locate position of a long helix in an RNA molecular envelope. Second, sugarphosphate backbones of nucleic acids are very sensitive to X-ray scattering and are very useful to delineate RNA molecular envelope $[13,14]$. 
A widely used modeling approach is to derive the information content in SAXS by generating a molecular envelope using software by Svergen and coworkers [15-18]. This is accomplished by laying out hundreds of identical beads (dummy atoms) in an enclosed volume and iteratively moving one at time in order to improve the fit between the SAXS profile of the model and the experimental data. The envelope is subsequently used for manual fitting of the helical segments in search of the global fold. Such an approach has been applied to derive topological folds of several RNAs [19-21]. In addition to constructing a molecular envelope, a complete SAXS spectrum may be used to determine the topological fold in two different ways. First, the SAXS-data fit may be used to filter [22] the candidate models obtained from other computational modeling programs. Alternatively, the SAXS-data fit can be used to efficiently sample the folding landscape starting from a simple 3D model of the secondary structural elements. The latter method can be accomplished through a fragment assembly search [23] or through "natural" hierarchical moves that follow from the modular architecture of RNAs (Y. R. Bhandari, J. R. Stagno, Y. -X. Wang unpublished work). With virtually no size limitation, and the ability to collect data under near-physiological conditions, SAXS can provide useful outlines of the global shape, which can be leveraged by computational methods to determine 3D topological folds of RNAs.

\section{Theory and Applications}

\subsection{Pure computational modeling}

Computational modeling of RNA folding is carried out by applying Newtonian mechanics (molecular dynamics simulations), Monte Carlo moves, or any other sampling 
algorithm. Explicit all-atom molecular dynamics (MD) simulations demand a significant amount of computational time even with the latest generation computers, and thus, in this context, may only be applicable for structure refinements. However, RNAs are predominantly comprised of A-form-like duplexes, and a survey of duplexes taken from RNA crystal structures in the PDB showed that the backbone atoms (phosphate and sugar) fluctuated to within only 1 A root mean squared deviation (rmsd) compared to their average positions [24]. Therefore, the computational cost of MD simulations can be greatly reduced to a first approximation by using a "reduced model" approach. This is generally true for an RNA molecule that is not undergoing large amplitude of local motion. For example, Ding et al. $[6,25,26]$ represented each nucleotide as three beads (sugar, phosphate, and base) and input RNA sequences to a discrete MD engine to predict their structures and folding dynamics. Their software, iFoldRNA [6], is mostly applicable to smaller $(<50 \mathrm{nt})$ RNAs. However, with the inclusion of additional constraints, a newer version, iFoldRNA-v2 [26], was able to improve the prediction accuracy of the 3D fold of the M-box riboswitch (161 nt; PDB ID 3PDR ) from $32 \AA$ to $7.7 \AA$ rmsd compared to the crystal structure. The use of any long-range contact information that can be obtained from biochemical assays may be valuable in improving the accuracy of a model.

Another successful technique for computing RNA models is the fragment assembly approach. The MC-Fold | MC-Sym software [5] uses nucleotide cyclic motifs (NCMs), which include variable-length lone-pair loops and double-stranded motifs from the structure database, to build a fragment library. The software searches for a suitable combination of NCMs for the input RNA sequence by assigning energy scores, and then assembles the 3D fragments corresponding to each NCM using a sampling algorithm. 
Prediction results of MC-Sym for thirteen RNAs (<50 nt) were less than $3.5 \AA$ rmsd compared to their experimentally determined structures. Although structure prediction by MC-Sym is limited to small RNAs with relatively simple folds, this modeling program is valuable in generating 3D models of larger RNAs, which can then be filtered by other methods using experimental SAXS data [19, 22].

Although structure databases contain several unique RNA folds, automated 3D structure prediction programs are mostly limited by molecule size and the availability of commonly occurring structural motifs. Therefore, interactive RNA modeling may be useful when it comes to more complex RNA folds involving multiple junctions, base triplets, highly convoluted pseudoknots, or unusually long internal or terminal loops. Using the RNA secondary structure as input, the RNA2D3D software [27] is useful for generating a simple 3D model and manipulating single strands, base pairs, and tertiary constraints. In addition, longer RNAs with greater structural complexity can be assembled using the popular programs, MANIP [28] and NAB [29].

\subsection{Modeling aided by SAXS data}

\subsubsection{Using a SAXS-derived molecular envelope}

To delineate the structural information contained in SAXS data of larger RNA molecules, it may be worthwhile to perform additional SAXS experiments on smaller, stable fragments. Choice of the fragment constructs is mostly by trial and error, guided by comparison of SAXS-derived envelopes of fragment constructs with that of the intact molecule. SAXS-derived molecular envelopes of domains and subdomains are likely to be more easily interpretable in terms of model fitting or identifying structural features, 
such as coaxial stacking. Such an approach is particularly useful because of modular nature of RNA molecules. The Divide-and-Conquer approach, which represents the manual fitting of individual SAXS envelopes of the fragments into that of the complete structure, features an additional benefit of SAXS in determining the topological folds of some unique RNAs [19-21, 30, 31]. As an example, Fang et. al. [21] used SAXS to determine the global fold of the HIV-1 Rev response element (RRE) RNA, which provided the structural basis for the specificity of viral RNA recognition. The study revealed an "A-shaped" structure, with a separation distance $(\sim 55 \AA)$ across the two legs coinciding approximately with that between the two arginine rich motifs in the Rev dimer (Figure 2) [32, 33].

A hybrid strategy can be applied for topological structure determination of RNA [19], which combines experimental SAXS data and computational software to generate structural models that best fit the SAXS envelope. Ali and coworkers [19] have studied the unliganded state of the TPP riboswitch using this hybrid approach. Riboswitches are regulatory elements of mRNA that undergo a conformational switch upon binding of small-molecule metabolites. Riboswitches in ligand-free conformations are flexible, and thus challenging for structural investigation. In the above study, three truncation mutants, which constituted the complete TPP riboswitch, were used for SAXS data collection (Figure 3). For each fragment, a number of structural models were produced using the MC-sym [5] software, and the model that best fit the SAXS-derived envelope of that fragment was selected. The fragment models were then combined in all possible ways to generate ensembles of the RNA, which were subsequently ranked based on their fit to the molecular envelope derived from the SAXS data of the whole riboswitch (Figure 3). 


\subsubsection{Using SAXS data as a filter}

One of the many utilities of SAXS-based modeling is the selection of candidates whose back-calculated SAXS profiles are consistent with experimental SAXS data. As an example, Yang et al. [22] developed a method called Fast-SAXS-RNA to filter the decoy models generated by MC-Sym using $\chi^{2}$ (goodness of fit to SAXS data), explicit treatment of the solvent layer, and a two-bead (backbone and base) model. The Fast-SAXS-RNA method was successful at calculating topological folds of tRNA ${ }^{\mathrm{val}}$ and the group I intron P4-P6 domain to $8.6 \AA$ and $16.0 \AA$ rmsd, respectively, compared to their native folds (Figure 4). In yet another study, Magdalena and coworkers [34] applied coarse graining of RNA to one-point-per-residue to model larger RNAs with MD simulations using the NAST software. The models predicted by NAST were filtered using simulated SAXS data and solvent accessibility (tertiary constraints) to generate $8.0 \AA$ and $16.3 \AA$ rmsd models, respectively, for tRNA ${ }^{\text {phe }}$ and the group I intron P4-P6 domain (Figure 4). It is interesting to note here the similarity in rmsd for both the tRNA $(\sim 8.0 \AA)$ and the P4-P6 domain $(\sim 16.0 \AA)$ models determined using two different methods. Results of both FastSAXS-RNA and NAST methods may, however, be biased by the limited availability of RNA structures to derive the constraints used for modeling the initial decoy structures.

\subsubsection{Conformational sampling by fitness to SAXS data}

As SAXS data contains the structural information on global fold, fitness to experimental SAXS data is a valuable guide for sampling 3D models of RNAs at the secondary structure level. This is accomplished through discrete sub-conformational changes (moves) aimed at minimizing the $\chi^{2}$ value obtained for each successive global 
conformation. One way to bring about the sub-conformational changes is to use the Monte-Carlo-based fragment assembly method, which replaces secondary-structure elements with matching fragments from a variable-length fragment library. This method, reported by Michal and coworkers, was successfully applied to RNAs ( $<70 \mathrm{nt}$ ) with relatively simple folds [23]. In contrast, RS3D (Y. R. Bhandari, J. R. Stagno, Y. -X. Wang unpublished work), combines secondary structure information and SAXS data to determine RNA topological folds through "natural" hierarchical moves satisfying the Monte Carlo Metropolis Test. Here, conformational sampling is carried out through independent rotations or translations at both the local (nucleotide) and global (duplexes, loops, junctions, or strategic combination thereof) levels. The program is also capable of utilizing any available long-range structural information as an additional filter to narrow down the best conformational states.

The allowed move sets in RS3D follow a decision-making algorithm that ensures agreement between the back-calculated and experimental SAXS profiles, and conformity to the secondary structure constraints. The scattering profile is calculated through pairwise interactions of the atomic form factors using the Debye equation [35]. To ease the computational overhead due to calculations involving all-atom interactions, a reduced model is adopted, which treats each nucleotide as a single unit, or "glob." The scattering form factor for a glob is calculated as

$$
F_{\text {glob }}(q)=\left[\sum_{i=1}^{M} \sum_{j=1}^{M} f_{i}(q) f_{j}(q) \frac{\sin \left(q r_{i j}\right)}{q r_{i j}}\right]^{1 / 2}
$$


In equation $\mathbf{1}, f_{i}$ and $f_{j}$ represent the atomic $\mathrm{X}$-ray form factors, $q$ is the momentum transfer in inverse angstroms, $r_{i j}$ is the pairwise distance between the $i^{\text {th }}$ and $j^{\text {th }}$ atom, and $M$ represents the number of atoms in the glob. Average glob scattering factors specific to each nucleotide were calculated from over 30 different high-resolution (better than $3 \AA$ ) crystal structures of RNA. Figure 5 shows a comparison of the all-atom scattering profile (red) plotted against the scattering profile derived from the glob form factors (blue) after applying the correction factors corresponding to each momentum transfer (q). Hence, the glob parameters are sufficient to generate a scattering profile that closely approximates the all-atom derived scattering curve in a coarse-grain calculation.

The initial step in RS3D is to input the secondary structure information into RNA2D3D [27] to construct an initial 3D model in an open conformation (simply satisfying the secondary structure lay out). Next, the initial model is coarse-grained to represent each nucleotide as a glob located at the center of its electron density. Distance restraints that characterize covalent connectivity, duplex formation, and helical stacking are fed into the algorithm as pre-stored values. Any additional topological constraints, if available, are also provided as input at this stage. The program then carries out independent moves, each of which involves either a translation or rotation of a complete helical unit, or a group of globs selected arbitrarily by a random number generator. Every new move in the search for the target structure is directed towards the minimization of $\chi^{2}$, which is calculated as,

$$
\chi^{2}=\frac{1}{N_{q}-1} \sum_{k=1}^{N_{q}}\left[\frac{I_{\text {expt }}\left(q_{k}\right)-g_{k} I_{\text {calc }}\left(q_{k}\right)}{\sigma\left(q_{k}\right)}\right]^{2}
$$


In equation 2, $N_{q}$ represents the total number of data points, $I_{\text {expt }}$ is the experimental scattering intensity, $g_{k}$ is the glob correction factor, $I_{c a l c}$ is the back calculated scattering intensity of the model structure using the Debye equation, and $\sigma\left(q_{k}\right)$ is the experimental noise. The $\chi^{2}$ values for both the current $\left(\chi_{\text {old }}^{2}\right)$ and new conformation $\left(\chi_{\text {new }}^{2}\right)$ are calculated, and the difference $\left(\chi_{\text {new }}^{2}-\chi_{\text {old }}^{2}\right)$ is subjected to the Metropolis test [36] given below.

$$
P_{\chi^{2}}= \begin{cases}e^{-\frac{\Delta \chi 2}{T}} & \text { if } \Delta \chi 2>0 \\ 1 & \text { otherwise }\end{cases}
$$

In equation 3, $\Delta \chi 2=\chi_{\text {new }}^{2}-\chi_{\text {old }}^{2}$ and $T$ represents the temperature factor used for applying simulated annealing every fixed number of iterations. The Metropolis test and the secondary structure constraints must be satisfied for each accepted new conformation, while the tertiary structure constraints act as a guide to converge to the native state. For each RNA, 10000 to 30000 conformations were generated by RS3D and subjected to further analysis. The degeneracy in SAXS data makes it challenging to filter the best possible candidate solely based on the $\chi^{2}$ value. Therefore, we ascribe two additional attributes to each conformation, namely, the solvent accessible surface area (SASA) and the degree of extendedness. The degree of extendedness $(\mathrm{E})$ is measured as,

$$
\mathrm{E}=\frac{D_{\max }}{3 *(N / 2)}
$$


In equation 4, $D_{\max }$ is the maximum particle dimension, and $N$ represents the total number of nucleotides present in the RNA. RNAs with two-way junctions and $\mathrm{E}>0.82$, as well as RNAs with three-way and higher junctions and $\mathrm{E}>0.7$, were considered to be in extended conformation. Deviations in $\mathrm{E}(\Delta \mathrm{E})$ of the calculated structures from the target structures were also computed for all conformations. If the molecule was not in extended conformation, the minimum sum of $\chi^{2}$ and SASA gave the best candidate, while, if the molecule was in extended conformation, the minimum sum of $\chi^{2}$ and $\Delta \mathrm{E}$ gave the best candidate.

RS3D was successfully applied to benchmark over 15 different RNAs that represent a majority of the folding architecture currently present in the structure database. Figure 6 shows the lowest rmsd topological folds of two RNAs, namely, adenine riboswitch and the MLV readthrough pseudoknot, determined to $4.8 \AA$ and $4.4 \AA$, when aligned against the protein data bank structures with codes 1Y26 [37] and 2LC8 [38], respectively. A calculated structure with this degree of accuracy would be expected to provide important structural information. For adenine riboswitch RNA, a long range tertiary interaction $(\sim 10 \AA)$ between the two apical loops, predicted from covariation analysis [39], was used as an additional tertiary restraint. The conformational sampling of the readthrough pseudoknot RNA was restrained by a pseudoknot predicted from mutational analysis $[40,41]$. The performance of RS3D in determining the topological folds is comparable to other computational softwares (Fast-SAXS-RNA, NAST) that utilize SAXS data. 


\section{Conclusion and outlook}

X-ray crystallography and NMR spectroscopy are still the methods of choice for high-resolution structure determination of RNAs. However they are often severely limited by technical challenges. Provided a limited number of high-resolution RNA structures, alternative methods that involve utilizing more easily accessible experimental data might be a practical way to obtain RNA 3D topological information. As SAXS experiments are carried out in solution, and RNA backbone atoms are good scatterers of X-rays, SAXS is highly suited and effective for RNA structure determination. The secondary structure of RNA can be correlated to the 3D folding. Therefore, native-like topological folds can be achieved through efficient conformational sampling that involves natural "hierarchical" moves of the secondary structure motifs, guided by SAXS data. Although the high spatial resolution can be achieved by extending measurement to the wide angle region with momentum transfer up to $2.51 / \AA$, which correspond to $\sim 2.5 \AA$, such number is convoluted and can not be directly used for resolution enhancement. Nevertheless, the spatial uncertainty in the structure determination using SAXS may be resolved by the inclusion of a few tertiary constraints, which are shown to significantly improve RNA modeling results to produce biologically significant topological folds $[26,34]$. Such information is routinely attainable. For example, a phylogenetic approach involving sequence alignment and covariational analysis may help predict some tertiary interactions. Furthermore, biochemical assays 
involving chemical mapping [42-44] and mutational analysis may also be used to obtain nucleotide-level tertiary contacts.

It is noteworthy to point out that the application of SAXS for RNA molecular modeling is generally based on an implicit assumption that an RNA molecule is not undergoing large amplitude motion on a millisecond to second time scale on which SAXS data is recorded and can then be treated as a rigid body. For an RNA molecule undergoing large motions, such an assumption is no longer true. In such cases, the SAXS data can no longer be interpreted by a single model and the envelope calculation does not converge, as being reflected in a very high normalized spatial discrepancy $[45,46]$. Consequently, SAXS data can only be interpreted using ensemble approach [21, 47, 48].

\section{Acknowledgments}

This work was supported by the Intramural Research Programs of the National Cancer Institute. The content of this publication does not necessarily reflect the views or policies of the Department of Health and Human Services, nor does mention of trade names, commercial products, or organizations imply endorsement by the US government. 


\section{References}

[1] J.S. Mattick, I.V. Makunin, Hum Mol Genet, 15 Spec No 1 (2006) R17-29.

[2] J.L. Rinn, H.Y. Chang, Annual review of biochemistry, 81 (2012) 145-166.

[3] E.A. Doherty, J.A. Doudna, Annu Rev Biophys Biomol Struct, 30 (2001) 457-475.

[4] D.E. Draper, Rna, 10 (2004) 335-343.

[5] M. Parisien, F. Major, Nature, 452 (2008) 51-55.

[6] S. Sharma, F. Ding, N.V. Dokholyan, Bioinformatics, 24 (2008) 1951-1952.

[7] R. Das, D. Baker, Proceedings of the National Academy of Sciences of the United States of America, 104 (2007) 14664-14669.

[8] M.H. Bailor, A.M. Mustoe, C.L. Brooks, 3rd, H.M. Al-Hashimi, Current opinion in structural biology, 21 (2011) 296-305.

[9] K.E. Deigan, T.W. Li, D.H. Mathews, K.M. Weeks, Proceedings of the National Academy of Sciences of the United States of America, 106 (2009) 97-102.

[10] C.E. Hajdin, S. Bellaousov, W. Huggins, C.W. Leonard, D.H. Mathews, K.M. Weeks, Proceedings of the National Academy of Sciences of the United States of America, 110 (2013) 5498-5503.

[11] O. Glatter, O. Kratky, Small Angle X-ray Scattering, Academic Press 1982.

[12] Y.M. Lvov, V.I. Troitsky, L.A. Feigin, Mol Cryst Liq Cryst, 172 (1989) 89-97.

[13] X. Fang, J.R. Stagno, Y.R. Bhandari, X. Zuo, Y.X. Wang, Current opinion in structural biology, 30 (2015) 147-160.

[14] X. Zuo, J. Wang, Y.-X. Wang, in: P. Haris (Ed.) Biomolecular NMR Spectroscopy, IOS Press2011, pp. 457-467.

[15] P.V. Konarev, V.V. Volkov, A.V. Sokolova, M.H.J. Kochb, D.I. Svergun, Journal of Applied Crystallography, 36 (2003) 1277-1282.

[16] D.I. Svergun, J. Appl. Crystallogr., 25 (1992) 495-503.

[17] D.I. Svergun, Biophysical journal, 76 (1999) 2879-2886.

[18] V.V. Volkov, D.I. Svergun, J. Appl. Cryst., 36 (2003) 860-864.

[19] M. Ali, J. Lipfert, S. Seifert, D. Herschlag, S. Doniach, Journal of molecular biology, 396 (2010) 153-165.

[20] J. Lipfert, J. Ouellet, D.G. Norman, S. Doniach, D.M. Lilley, Structure, 16 (2008) 1357-1367.

[21] X. Fang, J. Wang, I.P. O'Carroll, M. Mitchell, X. Zuo, Y. Wang, P. Yu, Y. Liu, J.W. Rausch, M.A. Dyba, J. Kjems, C.D. Schwieters, S. Seifert, R.E. Winans, N.R. Watts, S.J. Stahl, P.T. Wingfield, R.A. Byrd, S.F. Le Grice, A. Rein, Y.X. Wang, Cell, 155 (2013) 594-605.

[22] S. Yang, M. Parisien, F. Major, B. Roux, The journal of physical chemistry. B, 114 (2010) 10039-10048.

[23] M.J. Gajda, D. Martinez Zapien, E. Uchikawa, A.C. Dock-Bregeon, PloS one, 8 (2013) e78007.

[24] J. Wang, X. Zuo, P. Yu, H. Xu, M.R. Starich, D.M. Tiede, B.A. Shapiro, C.D.

Schwieters, Y.X. Wang, Journal of molecular biology, 393 (2009) 717-734.

[25] F. Ding, S. Sharma, P. Chalasani, V.V. Demidov, N.E. Broude, N.V. Dokholyan, Rna, 14 (2008) 1164-1173.

[26] A. Krokhotin, K. Houlihan, N.V. Dokholyan, Bioinformatics, 31 (2015) 2891-2893. 
[27] H.M. Martinez, J.V. Maizel, Jr., B.A. Shapiro, J Biomol Struct Dyn, 25 (2008) 669683.

[28] C. Massire, E. Westhof, J Mol Graph Model, 16 (1998) 197-205, 255-197.

[29] W.A.S.-S. Tom Macke, Russell A. Brown, Istvan Kolossvary, Yannick Bomble and David A. Case, Website: http://casegroup.rutgers.edu/casegr-sh-2.2.html, 04/01/2016.

[30] C.P. Jones, W.A. Cantara, E.D. Olson, K. Musier-Forsyth, Proceedings of the National Academy of Sciences of the United States of America, 111 (2014) 3395-3400. [31] S.E. Tsutakawa, G.L. Hura, K.A. Frankel, P.K. Cooper, J.A. Tainer, J Struct Biol, 158 (2007) 214-223.

[32] M.D. Daugherty, B. Liu, A.D. Frankel, Nature structural \& molecular biology, 17 (2010) 1337-1342.

[33] M.A. DiMattia, N.R. Watts, S.J. Stahl, C. Rader, P.T. Wingfield, D.I. Stuart, A.C. Steven, J.M. Grimes, Proceedings of the National Academy of Sciences of the United States of America, 107 (2010) 5810-5814.

[34] M.A. Jonikas, R.J. Radmer, A. Laederach, R. Das, S. Pearlman, D. Herschlag, R.B. Altman, Rna, 15 (2009) 189-199.

[35] P. Debye, Annalen der Physik, 351 (1915) 809-823.

[36] N. Metropolis, Rosenbluth, A. W., Rosenbluth, M. N., Teller, A. H., and Teller, E., The Journal of Chemical Physics, 21 (1953) 1087-1092.

[37] A. Serganov, Y.R. Yuan, O. Pikovskaya, A. Polonskaia, L. Malinina, A.T. Phan, C. Hobartner, R. Micura, R.R. Breaker, D.J. Patel, Chemistry \& biology, 11 (2004) 17291741.

[38] B. Houck-Loomis, M.A. Durney, C. Salguero, N. Shankar, J.M. Nagle, S.P. Goff, V.M. D'Souza, Nature, 480 (2011) 561-564.

[39] M. Mandal, B. Boese, J.E. Barrick, W.C. Winkler, R.R. Breaker, Cell, 113 (2003) 577-586.

[40] N.M. Wills, R.F. Gesteland, J.F. Atkins, EMBO J, 13 (1994) 4137-4144.

[41] S.L. Alam, N.M. Wills, J.A. Ingram, J.F. Atkins, R.F. Gesteland, Journal of molecular biology, 288 (1999) 837-852.

[42] C. Waldsich, Methods in Molecular Biology, (2014).

[43] C.Y. Cheng, http://biorxiv.org/content/early/2014/04/25/004556 (preprint link), (2014).

[44] P.J. Homan, A. Tandon, G.M. Rice, F. Ding, N.V. Dokholyan, K.M. Weeks, Biochemistry, 53 (2014) 6825-6833.

[45] D. Franke, D.I. Svergun, J. Appl. Cryst., 42 (2009) 342-346.

[46] M.B. Kozin, D.I. Svergun, Journal of Applied Crystallography, 34 (2001) 33-41.

[47] P. Bernado, E. Mylonas, M.V. Petoukhov, M. Blackledge, D.I. Svergun, Journal of the American Chemical Society, 129 (2007) 5656-5664.

[48] C.D. Schwieters, G.M. Clore, Biochemistry, 46 (2007) 1152-1166. 


\section{Figure Captions}

Figure 1. Application of SAXS data into 3D modeling of RNAs. Image for the divide- $\&$ conquer approach is adopted from reference [21]

Figure 2. Application of the "divide-and-conquer" approach to RRE RNA [21]. SAXS density maps reconstructed from smaller constructs are manually fitted into the envelope representing the full length shown at three different views on the bottom.

Figure 3. (left) Secondary structure of complete TPP riboswitch. (middle) Secondary structure of three fragment constructs. (right) Best ranked combined model fitted inside the molecular envelope of the complete TPP riboswitch in unliganded state [19].

Figure 4. a) Ribbon representation of tRNA (top) and P4-P6 domain (bottom) of group I intron, solved by MC-Sym and SAXS fit filtering at $8.6 \AA$ and $16.0 \AA$ rmsd compared to the database structures in red [22]. b) Best ranked clusters of corresponding RNAs solved by NAST using tertiary constraints at $8.0 \AA$ and $16.3 \AA$ average rmsd compared to the database structures [34]. The corresponding similarity in rmsd is interesting to note.

Figure 5. Comparison of scattering profile calculated from all atom (blue) against glob calculation (red). The number of nucleotides in each RNA in represented by N.

Figure 6. a) Left panel shows the lowest rmsd (4.9 ̊) structure (blue) of adenine riboswitch RNA computed by RS3D, and aligned against the crystal structure (PDB ID 
$1 Y 26$, red). The right panel shows an ensemble of lowest scored $\left(\chi^{2}+\right.$ SASA) top 10 structures including the lowest rmsd conformation, aligned against the crystal structure. The longest junction (8 $\mathrm{nt})$ and apical loop regions are disordered due to lack of additional restraints. The conformation in green is an outlier. b) Left panel shows the lowest rmsd (4.4 A) structure (blue) of MLV readthrough pseudoknot RNA computed by RS3D, and aligned against the NMR structure (PDB ID 2LC8, red). The right panel shows an ensemble of lowest scored $\left(\chi^{2}+\right.$ SASA $)$ top 10 structures including the lowest rmsd conformation, aligned against the crystal structure. A single stranded region (13 nt) connecting to the 3 ' end through a pseudoknot is disordered due to lack of additional restraints. 


\section{SAXS Data}

Figure 1 


\section{II-III-IV}

Secondary structures
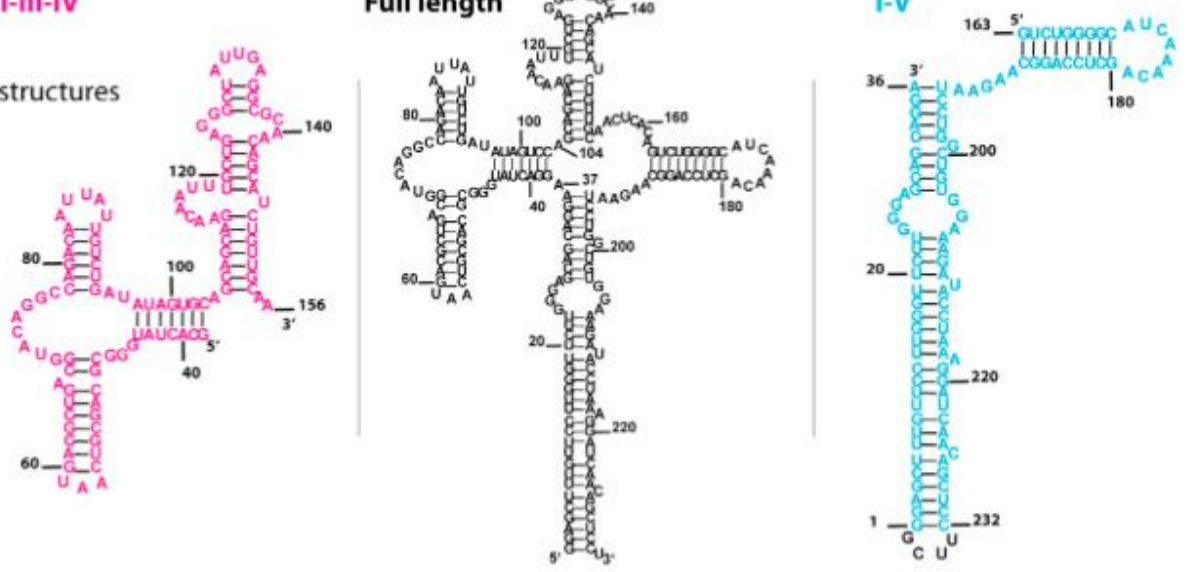

Envelopes

Superimposition

\section{Figure 2}

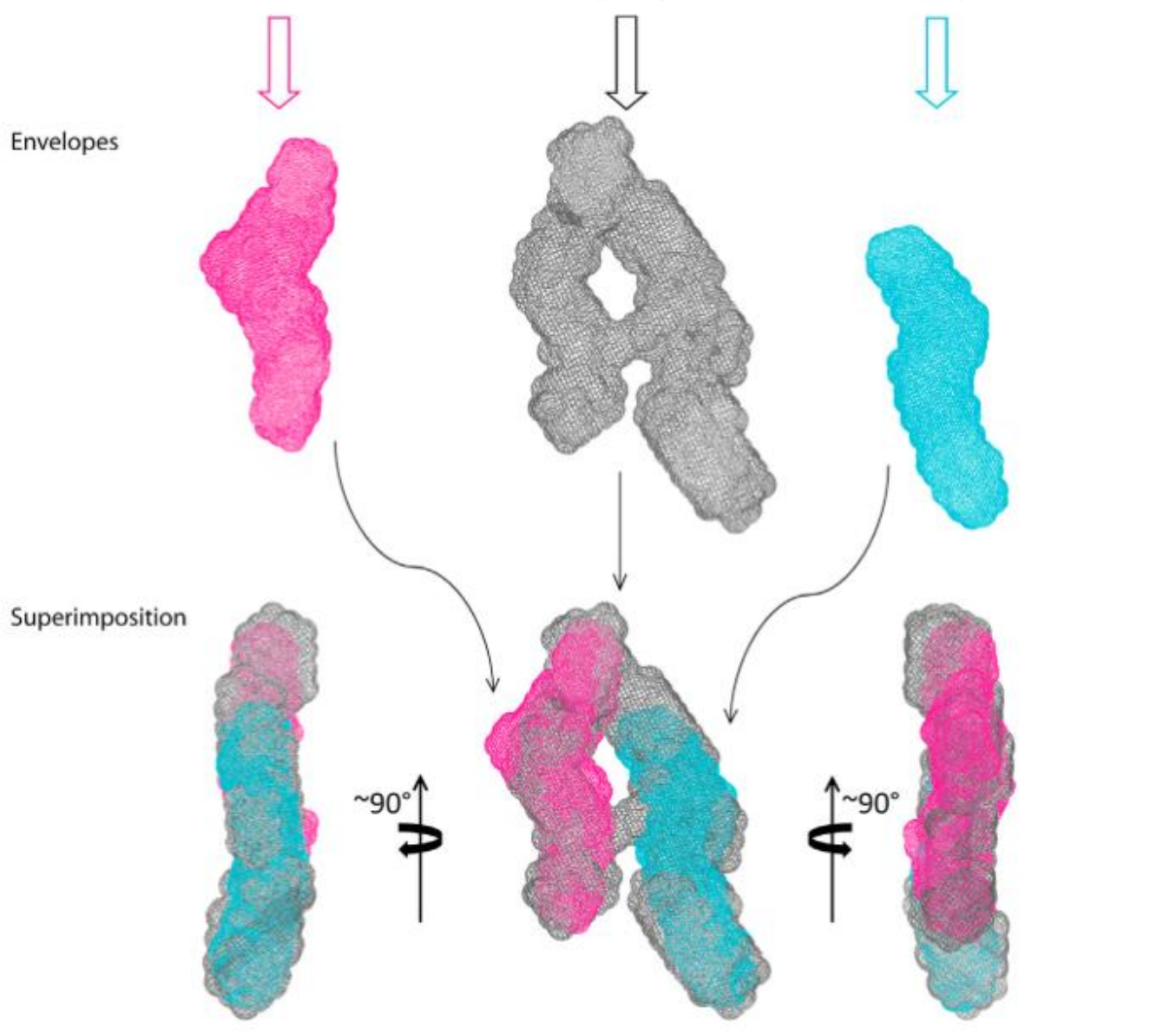




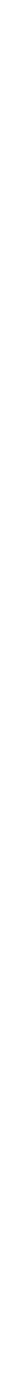

Figure 3 
a)
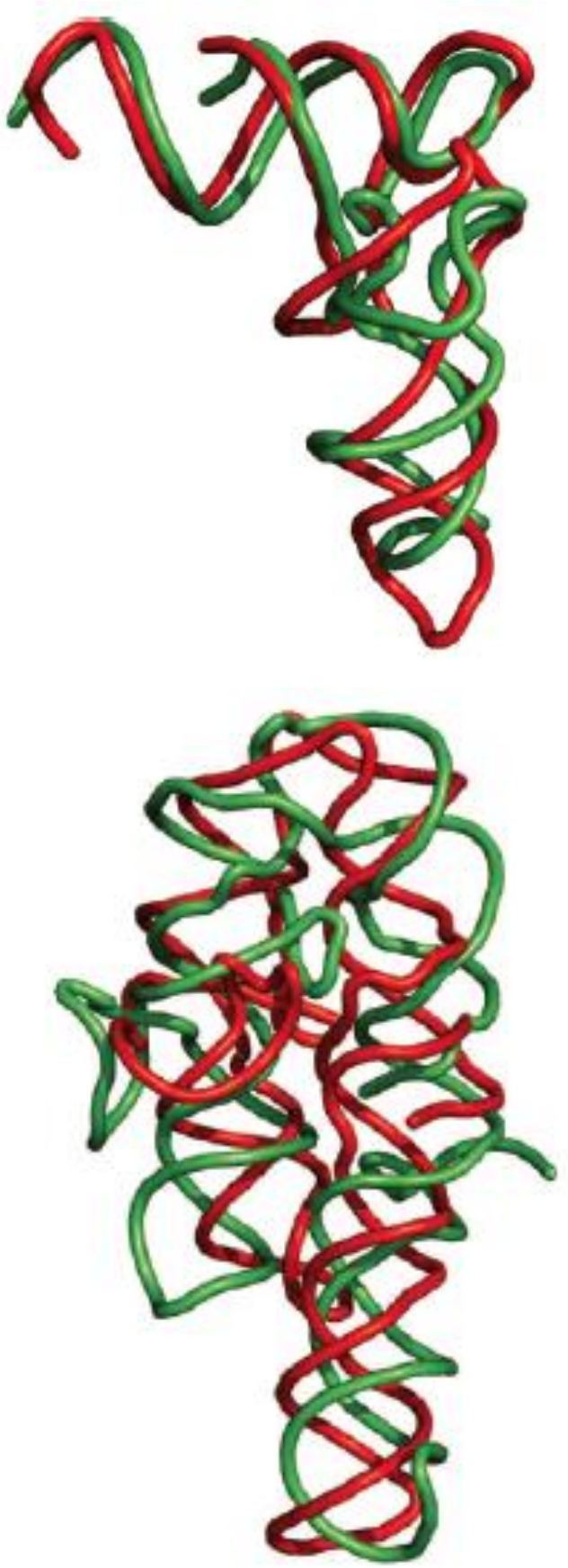

b)
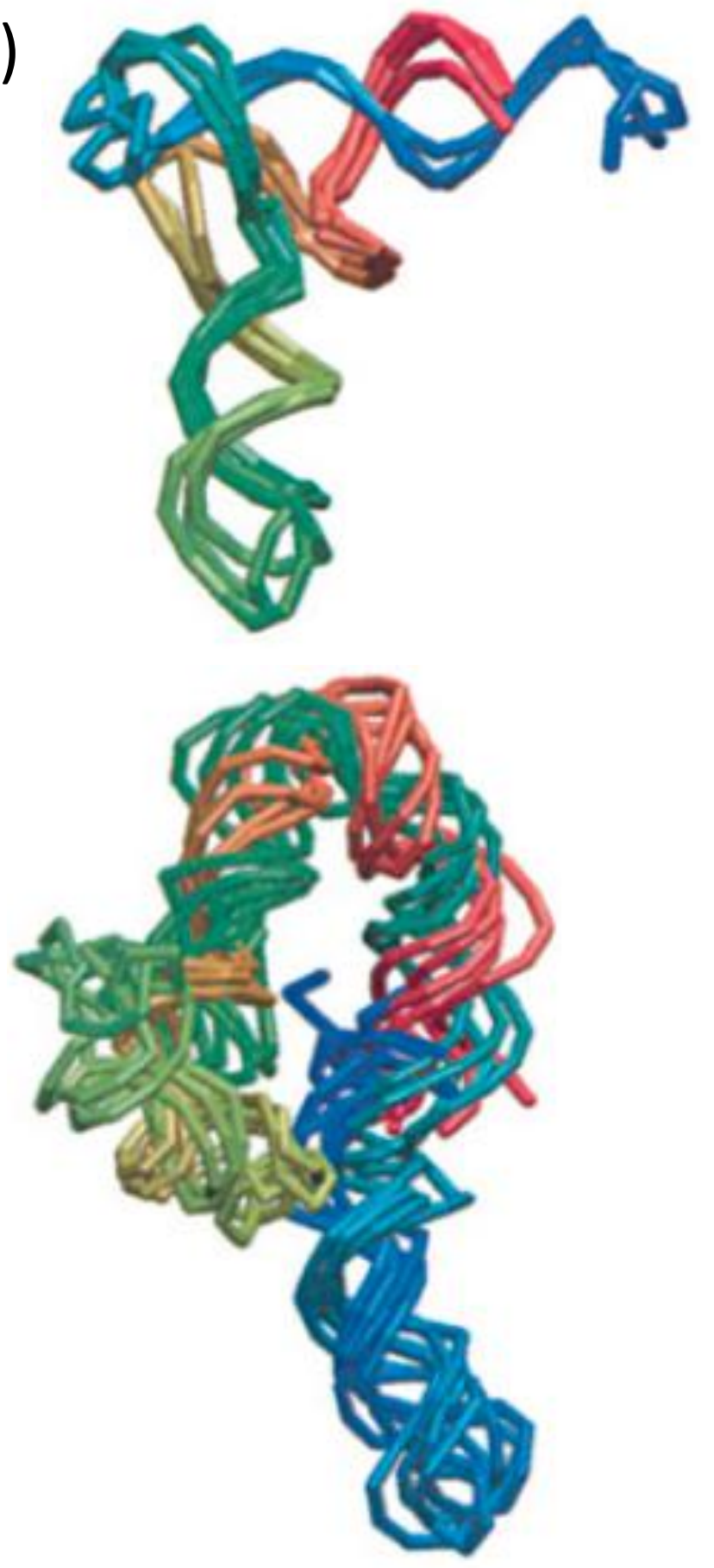

Figure 4 
a)
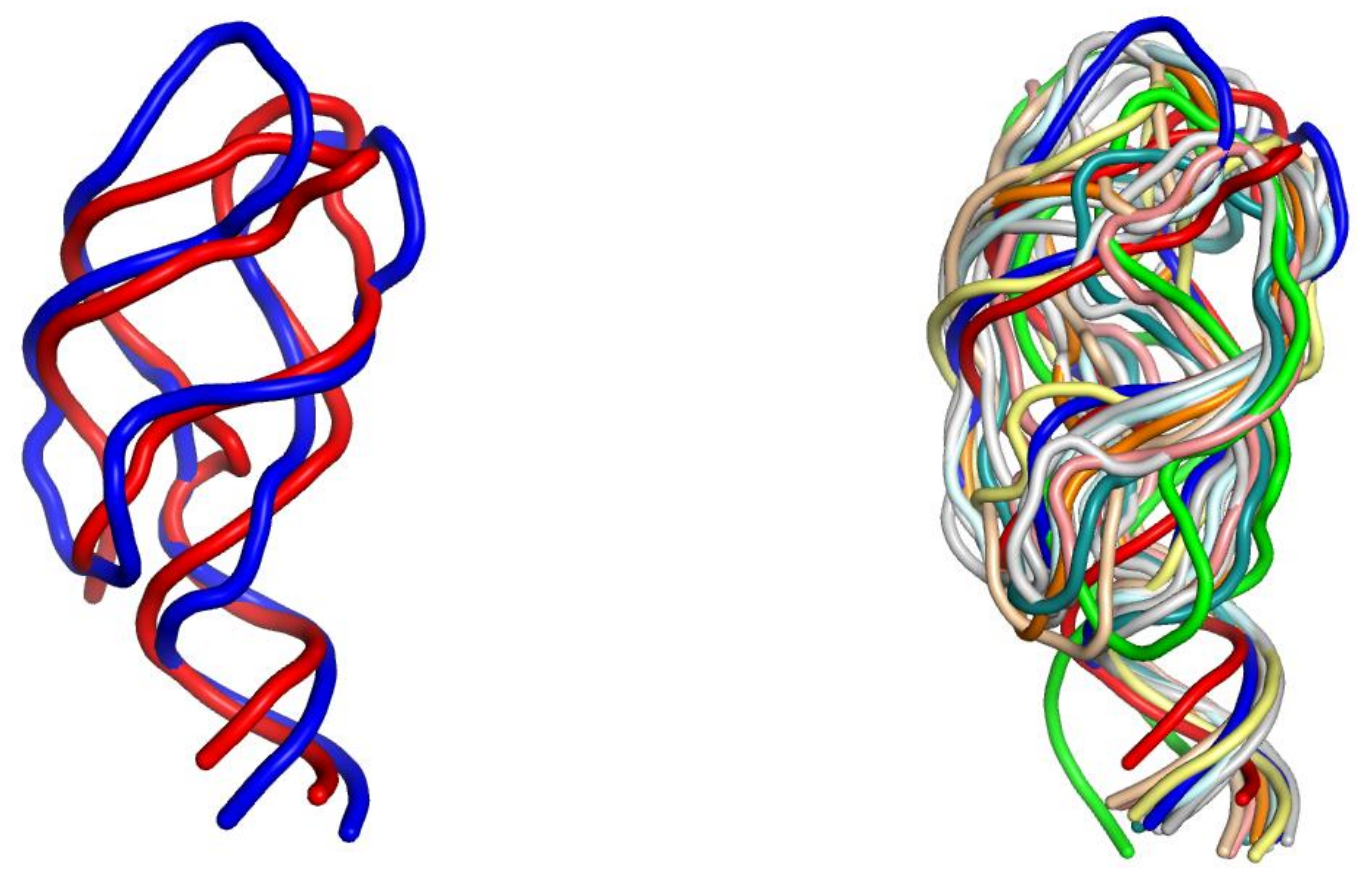

b) Figure 6
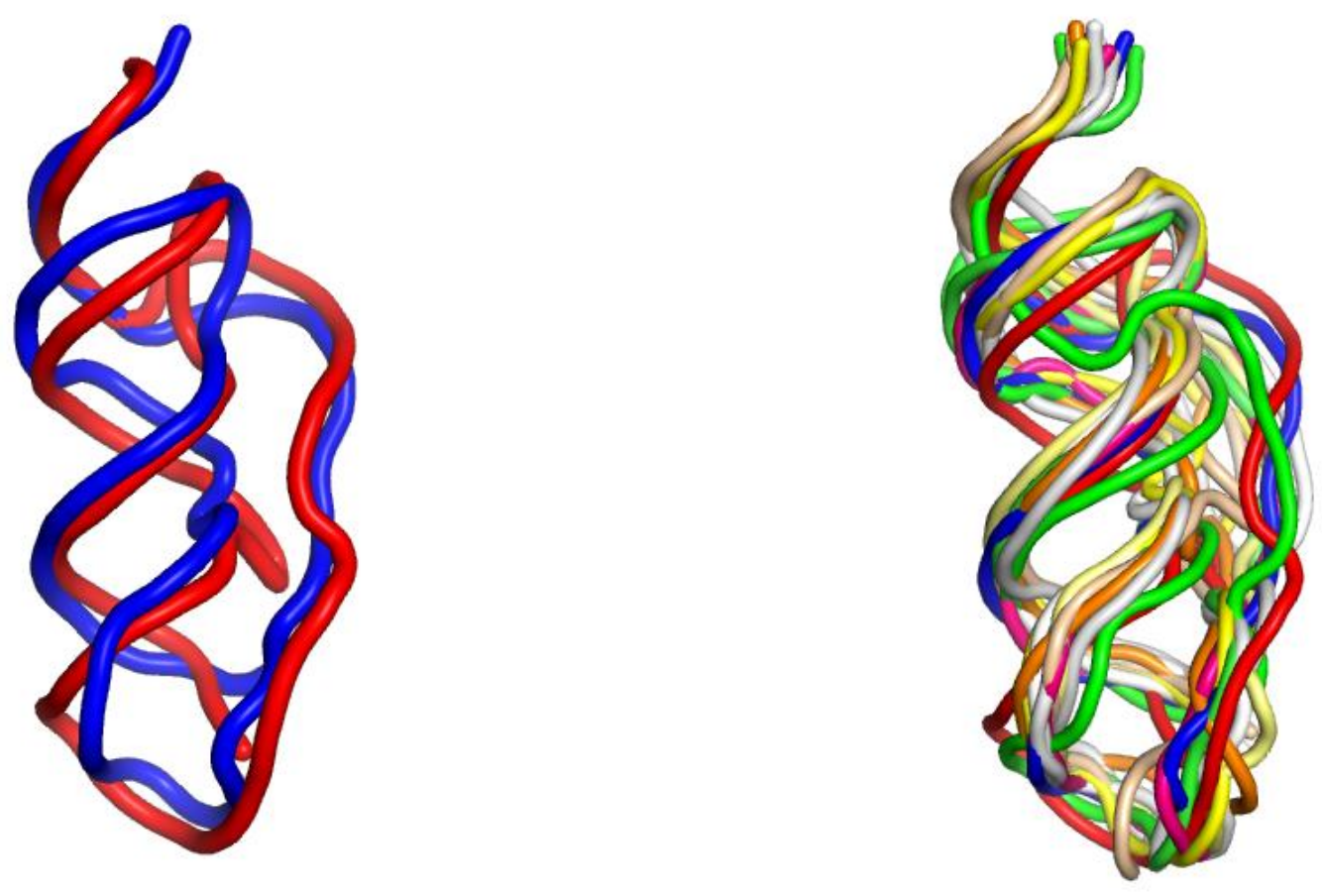